\title{
EL SISTEMA DE PALANCAS BASE PARA EL ANALISIS MECANICO DEL MOVIMIENTO CORPORAL HUMANO Y SUS ALTERACIONES.
}

\author{
Arcila. J, ${ }^{1}$, Cardona. D ${ }^{2}$, Becerra. $\mathrm{H}^{3}$
}

Juan Cancio Arcila Arango 1: Médico y cirujano, Licenciado en Matemáticas, Especialista en Gerencia en Servicios de Salud, Mg en Ciencias de la Actividad Física y el Deporte. Docentes de tiempo completo del Politécnico Colombiano Jaime Isaza Cadavid jcarcilaa@elpoli.edu.co

Donaldo Cardona Nieto2: Especialista, Mg y Doctor en Ciencias del Deporte, Docentes de tiempo completo del Politécnico Colombiano Jaime Isaza Cadavid dcardona@elpoli.edu.co

Henry Alfonso Becerra Riaño ${ }^{3}$ : Fisioterapeuta, Especialista en Pedagogía Universitaria, Mg en Ciencias de la Actividad Física y el Deporte Fisioterapeuta, Especialista en Pedagogía Universitaria, Mg en Ciencias de la Actividad Física y el Deporte. Docente Universidad de Pamplona, Director Programa de Fisioterapia hybecerra@unipamplona.edu.co

\section{RESUMEN.}

En este artículo se parte de la definición de máquinas simples para abordar primero los conceptos físicos generales de las palancas, y luego la interpretación vectorial específica de las palancas anatómicas involucradas en los movimientos del cuerpo humano. Se muestra una perspectiva analítica de los diferentes componentes morfológicos anatómicos y se establece una relación con sus equivalentes físicos biomecánicos conectados conceptualmente por la Fisiología articular. la identificación de los fulcros y de los puntos críticos de aplicación de las fuerzas que permite caracterizar los géneros de palanca, así como los factores que, dependiendo de la actitud postural asumida por los segmentos corporales implicados, condicionan en una misma articulación la utilización de diferentes tipos de palancas. Finalmente se presenta de manera esquemática una aproximación al entendimiento de la alteración biomecánica que se presenta en la conformación de un sistema de palancas cuando sus componentes morfológicos sufren alteraciones.

Palabras clave: Movimiento humano. Sistema de palancas anatómicas. Gesto articular. Análisis mecánico.

\section{ABSTRACT}

In this article we start with the definition of simple machines to first approach the general physical concepts of the levers, and then the specific vectorial interpretation of the anatomical levers involved in the movements of the human body. We present an analytical perspective of the different anatomical morphological components and establish a relation with their biomechanical physical equivalents conceptually connected by the articular Physiology. The identification of the fulcrums and the critical points of application of the forces that allow to characterize the lever genres, as well as the factors that, depending on the postural attitude assumed by the involved body segments, condition in a same articulation the use of different Types of levers. Finally, an approximation to the understanding of the biomechanical alteration that is presented in the conformation of a levers system is presented in a schematic way when its morphological components undergo alterations.

Keywords: Human movement. System of anatomical levers. Joint gesture. Mechanical analysis.

Convocatoria II trimestre. Recibido el 1 de Marzo de 2017; Aceptado el 1 de junio de 2017. 


\section{INTRODUCCIÓN}

Una máquina es un dispositivo conformado por varios elementos, unos móviles y otros fijos, los cuales actúan conjuntamente para utilizar energía o realizar un trabajo. "El principio fundamental de todas las máquinas es que la cantidad de trabajo obtenido no puede ser mayor que la energía que se les suministra" (MacDonald/Burns, 1989). Una máquina puede cambiar el módulo (valor numérico), la dirección o el método de aplicación de una fuerza para obtener algún provecho (Bueche, 1991). Una máquina en la que la energía es suministrada por una sola fuerza, de manera que realiza trabajo venciendo una sola resistencia, se denomina máquina simple. Muchos dispositivos que observamos en la vida cotidiana, tales como la palanca, el plano inclinado, la polea, la manivela y el gato, son máquinas simples. "Probablemente, ningún dispositivo mecánico es tan antiguo como la palanca; sencilla y versátil, no hay duda de que es más antigua que nosotros, los humanos" (Vogel, 2000).

Los movimientos humanos, desde los más simples hasta los más complejos, pueden interpretarse como el resultado de la confrontación de las fuerzas internas corporales con las fuerzas externas del medio ambiente. Un conjunto de elementos anatómicos (Tabla No 1) provisto de una sofisticación estructural, permite realizar una visión analógica que trasciende hacia la perspectiva funcional, fisiológica, que caracteriza los eventos biomecánicos del gesto articular. Las articulaciones, entendidas como el sitio de unión de dos o más huesos (Calais-Germain, 2010), "son los engranajes que mantienen unidos entre sí a los elementos óseos, permitiéndoles llevar a cabo los movimientos para los cuales están dispuestos" (Fucci y cols., 1998). Las articulaciones son, entonces, los fulcros 0 apoyos de las máquinas anatómicas del cuerpo humano. Los huesos maduros son estructuras relativamente rígidas, son los elementos constitutivos de los segmentos corporales que son objeto del desplazamiento, las palancas que esperan ser movilizadas. La máquina molecular que cumple con tal propósito es el músculo, un dispositivo que convierte la energía química orgánica proveniente de los alimentos (Enoka, 2002), en energía mecánica de tipo cinético para la contracción muscular.
Así, el músculo actúa como un transductor y, en consecuencia, es el motor que genera el movimiento articular. Los tendones son estructuras encargadas de trasmitir directa 0 indirectamente la tracción muscular a los huesos en los que se insertan (Guillen y cols., 2002), comportándose así, como cables de transmisión de fuerzas. Los tendones, junto con las cápsulas y los ligamentos, son estructuras pasivas que actúan como estabilizadores articulares secundarios. Específicamente, la cápsula está formada por tejido conectivo denso que rodea los extremos articulares de los huesos que intervienen en la articulación, incluyendo los elementos intra-articulares sobre los cuales ejerce una labor mecánica de protección y contención, con delimitación espacial de su contenido. Los ligamentos al conectar segmentos óseos contiguos guiando el movimiento y evitando desplazamientos excesivos, actúan como refuerzos estabilizadores o cierres de seguridad de la articulación. Revistiendo internamente la articulación se proyecta desde la cápsula una estructura provista de vellosidades llamada membrana sinovial, la cual actúa como fuente encargada de proveer el líquido sinovial necesario para la nutrición del cartílago y lubricación de la articulación. El cartílago hialino que recubre las superficies articulares de los huesos en contacto y el cartílago fibroso presente en los discos intra-articulares, actúan como amortiguadores que redistribuyen las cargas y generan compensaciones hidromecánicas, con el fin de prevenir lesiones articulares y atenuar los procesos degenerativos como la artrosis.

\begin{tabular}{|l|l|}
\hline \multicolumn{2}{|c|}{ ESTRUCTURA ARTICULAR } \\
\hline $\begin{array}{c}\text { PERSPECTIVA } \\
\text { ESTRUCTURAL }\end{array}$ & \multicolumn{1}{|c|}{$\begin{array}{c}\text { PERSPECTIVA } \\
\text { FUNCIONAL }\end{array}$} \\
\hline ANATOMICO & $\begin{array}{l}\text { INTERPRETACIÓN } \\
\text { MECÁNICO }\end{array}$ \\
\hline 1. Articulación. & $\begin{array}{l}\text { Fulcro: Apoyo: } \\
\text { Junta mecánica. }\end{array}$ \\
\hline 2. Hueso & Palanca \\
\hline 3. Musculo. & Motor, transductor \\
\hline 4. Tendon. & $\begin{array}{l}\text { Cable de de de d } \\
\text { transmisión de } \\
\text { fuerzas }\end{array}$ \\
\hline 5. Capsula. & Contenedor \\
\hline 6. Ligamento. & Cierre \\
\hline
\end{tabular}




\begin{tabular}{|l|l|}
\hline & $\begin{array}{l}\text { seguridad, } \\
\text { refuerzo. }\end{array}$ \\
\hline 7. Membrana sinovial. & $\begin{array}{l}\text { Fuente } \\
\text { dispensador }\end{array}$ \\
\hline 8. Liquido sinovial. & Aceite: Lubricante \\
\hline 9. Cartílago hialino & Amortiguador. \\
\hline $\begin{array}{l}\text { 10. Fibrocartilago. } \\
\text { (Discos, anillos } \\
\text { rodetes) }\end{array}$ & $\begin{array}{l}\text { Amortiguador, } \\
\text { acoplador. }\end{array}$ \\
\hline
\end{tabular}

Tabla N ${ }^{\circ}$ 1. Componentes anatómicos y equivalentes mecánicos para el gesto articular.

\section{ELEMENTOS DE UNA PALANCA ANATÓMICA.}

Una palanca está compuesta por tres elementos:

1. Una barra rígida sobre la que actúan las fuerzas, anatómicamente en el cuerpo humano corresponde a un hueso o segmento anatómico que se quiere movilizar. El peso de esta barra más el peso del objeto que se quiere movilizar constituye la carga a vencer (o levantar) llamada Resistencia $(R)$, la cual se aplica en el centro de gravedad o baricentro de barra más objeto (carga total) y, se dirige verticalmente hacia el centro de la tierra. Para los segmentos anatómicos el baricentro del segmento anatómico más objeto, se calcula de acuerdo con parámetros inerciales. De estos los más frecuentemente utilizados son los de localización del Centro de gravedad $(\mathrm{Cg})$ de los segmentos corporales propuestos por Clauser y col. en 1969, los ajustes de estos realizados por Hinrichs en 1990 y los de Leva en 1996, referenciados por Gutiérrez (1999).

Es importante tener en cuenta que al desarrollo de la resistencia también contribuyen los antagonistas y las fascias que se oponen al progreso del gesto en una determinada dirección.

2. Un punto respecto al cual gira la barra rígida, el cual anatómicamente en el cuerpo humano corresponde a la articulación. Este punto llamado "fulcro" o Apoyo (A), es el centro de rotación respecto al cual se realiza el gesto articular que mueve el segmento anatómico. Es importante tener en cuenta que dependiendo de la posición de la articulación en la ejecución del gesto, ocurren variaciones en la localización del centro de rotación y, por tanto, se trabaja con la "centroda" o centro de rotación instantáneo.

3. Una fuerza aplicada sobre la barra rígida, la que en el cuerpo humano es realizada por el músculo agonista en un gesto articular. Esta fuerza es llamada Potencia (P) y se aplica en algún punto de la barra. En las palancas anatómicas corresponde a la fuerza muscular aplicada en el punto de inserción donde se conecta el tendón al hueso. La fuerza muscular se representa gráficamente por un vector cuyo origen es el punto de inserción (punto de aplicación de la fuerza) y su dirección es el ángulo de tensión muscular. Este último corresponde a la relación espacial que hay entre el eje mecánico del hueso (o segmento anatómico) y la línea de acción muscular, la cual pasa por el punto de inserción y sigue la dirección del tendón. Adicionalmente, el eje mecánico que representa el segmento óseo, es una línea recta imaginaria que pasa por los centros de las superficies articulares ubicadas en los extremos, e incluye su centro de gravedad (Vandervael, 1961).

En el análisis de palancas es determinante la diferenciación de algunos parámetros mecánicos, a saber:

\section{Brazos de palanca}

Son las distancias medidas entre el fulcro donde está ubicado el eje de rotación de la palanca y los puntos de aplicación de las fuerzas (Donskoi, 1982).

\section{Brazos de fuerza}

Son las distancias medidas entre el fulcro donde está ubicado el eje de rotación de la palanca y las líneas de acción de las fuerzas (Donskoi, 1982). Así, estas son distancias perpendiculares.

No obstante, hay diversidad de definiciones de varios autores con respecto a estos términos. Como lo expresan Luttgens y Wells (1982), verdaderamente, un brazo de palanca es un brazo de momento, de tal forma que la distancia perpendicular entre el fulcro y la línea de fuerza del esfuerzo (o potencia) es, el brazo de 
esfuerzo del momento o brazo de esfuerzo y, de manera similar, la distancia perpendicular entre el fulcro y la línea de fuerza de resistencia es el brazo de resistencia del momento o brazo de resistencia. Estos son llamados por Gowitzke y Milner (1999) respectivamente brazo perpendicular de potencia o brazo de impulso de la potencia y brazo perpendicular de resistencia o brazo de impulso de la resistencia.

Vogel (2003), Gutiérrez (1999), Aguado (1993), Grosser et al. (1991), Rasch y Burke (1986), coinciden con las definiciones de Luttgens y Wells (1982).

Fucci y cols. (1998) y Guillén y Linares (2002) por el contrario, consideran como brazo de potencia, la distancia que hay entre el punto de apoyo y el punto de aplicación de la potencia; así, en las palancas anatómicas es la distancia entre la articulación y el punto de aplicación de la fuerza muscular. De manera similar, consideran comobrazo de resistencia la distancia que hay entre el punto de apoyo y el punto de aplicación de la resistencia; así, en las palancas anatómicas es la distancia entre la articulación y el baricentro del segmento.

En estas circunstancias, el torque de la fuerza muscular respecto a la articulación, puede calcularse multiplicando el brazo de potencia por el componente perpendicular de la fuerza muscular en el sistema de coordenadas convenido y teniendo presente el signo correspondiente. Análogamente, el torque del peso del segmento respecto a la articulación, puede calcularse multiplicando el brazo de resistencia por el componente perpendicular del peso del segmento en el sistema de coordenadas convenido y teniendo presente el signo correspondiente. En este trabajo se asumen las siguientes definiciones para los brazos de fuerzas:

Brazo de potencia (BP): Es la distancia perpendicular que hay entre el punto de apoyo y la línea de acción de la potencia. Así, en las palancas anatómicas es la distancia entre la articulación y la línea de acción de la fuerza muscular. El torque de la fuerza muscular respecto a la articulación, puede calcularse multiplicando el brazo de potencia por la fuerza muscular teniendo presente el signo correspondiente.
Brazo de resistencia (BR): Es la distancia perpendicular que hay entre el punto de apoyo y la línea de acción de la resistencia. Así, en las palancas anatómicas es la distancia entre la articulación y la línea de acción de la resistencia. El torque del peso del segmento respecto a la articulación, puede calcularse multiplicando el brazo de resistencia por el peso del segmento teniendo presente el signo correspondiente.

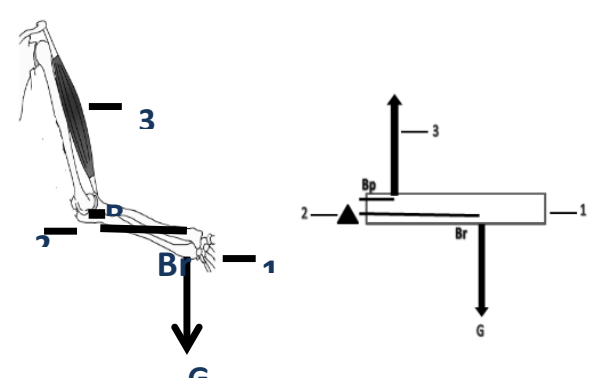

\begin{tabular}{|l|l|}
\hline \multicolumn{1}{|c|}{ A } & \multicolumn{1}{c|}{ B } \\
\hline 1. Hueso & 1. Palanca \\
\hline 2. Articulación & 2. Punto de apoyo. \\
\hline 3. Musculo & 3. Vector fuerza. \\
\hline 4. Gravedad. & 4. Vector Gravedad \\
\hline \multicolumn{2}{|c|}{ Bp: Brazo de palanca } \\
\hline \multicolumn{2}{|c|}{ Br : Brazo de resistencia } \\
\hline
\end{tabular}

Figura 2. Ejemplo de representación morfológica (A) y mecánica (B) de un sistema de palancas.

Teniendo en cuenta tales definiciones, resulta claro que la fuerza muscular no actúa, en general sobre las palancas óseas, en sentido perpendicular a los ejes mecánicos de los segmentos corporales y, en consecuencia, de acuerdo a los planteamientos de Hochmuth (1973), en tales casos el brazo de potencia es siempre inferior a la distancia entre el punto de apoyo y el punto de aplicación de la fuerza muscular. Basados en el hecho de que la función trigonométrica del seno de un ángulo (figura $\mathrm{N}^{0} 3$ ) puede tomar valores entre 0 y 1 , situación que se expresa por medio de la relación de orden $\mathbf{0} \leq \boldsymbol{s e n} \propto \leq$ 1 entonces ocurre que $0 \leq d$. $\operatorname{sen} \propto \leq \boldsymbol{d}$, asi que, $\boldsymbol{d} . \operatorname{sen} \propto \leq \boldsymbol{d}$ lo que significa matemáticamente que la distancia perpendicular (BP o BR) es menor o igual que la distancia entre los puntos de apoyo y de aplicación de 
fuerza $(P \circ R)$. En este sentido, la acción muscular sobre la palanca ósea resulta más eficaz para valores angulares cercanos a los $90^{\circ}$ (Hainaut, 1976). Como la fuerza muscular varía de forma directamente proporcional al seno del ángulo de inserción, el brazo de potencia es máximo cuando dicho ángulo vale $90^{\circ}$.

Los torques que se han mencionado son los que Vogel (2003) llama momentos de fuerza. Así, el torque de la potencia con respecto a la articulación es el momento de potencia y, el torque de la resistencia con respecto a la articulación, es el momento de resistencia.

"El torque o efecto rotatorio de una fuerza se representa por la letra griega $\boldsymbol{\tau}$ y se define matemáticamente como el producto de la fuerza por la distancia perpendicular entre la línea de acción de dicha fuerza y el punto que representa el centro de rotación. También puede definirse como el producto del componente de la fuerza que es perpendicular al eje mecánico de la palanca y la distancia entre el punto de aplicación de dicha fuerza y el punto que representa el centro de rotación. El torque es un vector perpendicular al plano formado por la fuerza $\boldsymbol{F}$ y la distancia $\boldsymbol{d}$ (Marion and Hornyak, 1982) cuyo signo obedece a la regla de la mano derecha 0 , simplemente, asumiendo como positivo el sentido anti-horario en el que el vector busca el centro de rotación por la vía más corta y, como negativo el sentido horario en el que el vector busca el centro de rotación por la vía más corta" (Arcila y cols., 2012). En general, para una fuerza oblicua ocurre lo siguiente:
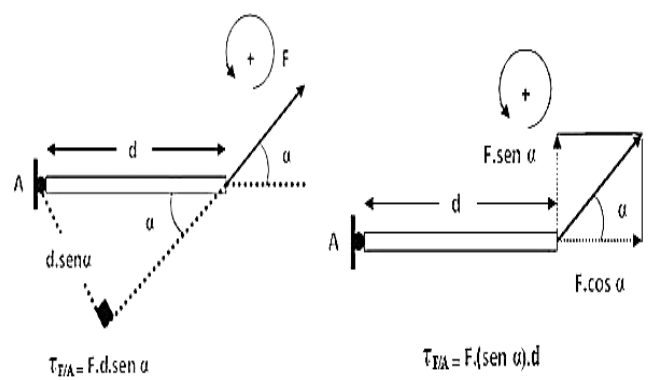

El símbolo $\boldsymbol{\tau} \boldsymbol{f} / \boldsymbol{a}$ se lee "el torque de la fuerza $F$ respecto al centro de rotación $A$ ".

Cuando la fuerza $F$ es perpendicular al eje mecánico de la palanca, se tiene que el ángulo $\propto=90^{\circ} y \operatorname{sen} 90^{\circ}=1$, es decir cuando el tendón del musculo tracciona sobre el hueso con un ángulo de $90^{\circ}$ se obtendrá el torque 0 momento máximo de la fuerza.

Observe que cuando la fuerza $F$ es oblicua, el torque es el mismo torque de su componente perpendicular al eje mecánico. Cuando la fuerza $F$ está sobre el eje mecánico de la palanca, se tiene que el ángulo $\alpha=$ $\mathbf{0}^{\circ} \boldsymbol{y} \operatorname{sen}^{\circ}=\mathbf{0}$ por lo que $\boldsymbol{\tau} \frac{f}{A}=\mathbf{0}$

Así que, todas aquellas fuerzas cuyas líneas de acción pasen por el centro de rotación, no producen torque" (Arcila y cols., 2012).

En el caso de una fuerza muscular oblicua que actúa sobre una palanca ósea, el componente perpendicular de dicha fuerza es denominado componente tangencial, porque es tangente a la trayectoria de los puntos de la palanca influyendo sobre su velocidad del movimiento, razón por la cual se le llama "de rotación" o "patente". A la componente paralela denominada normal, la cual presiona unas contra otras las superficies fijando la articulación se le llama "de reforzamiento" o "latente". (Zatsiorski, 1990).

Es importante tener en cuenta el conjunto total de fuerzas que actúan sobre una palanca: en la Figura 3 se ilustran las fuerzas que actúan sobre la palanca del eje antebrazo-muñecamano-carga externa en una flexión de codo por acción del músculo bíceps braquial.

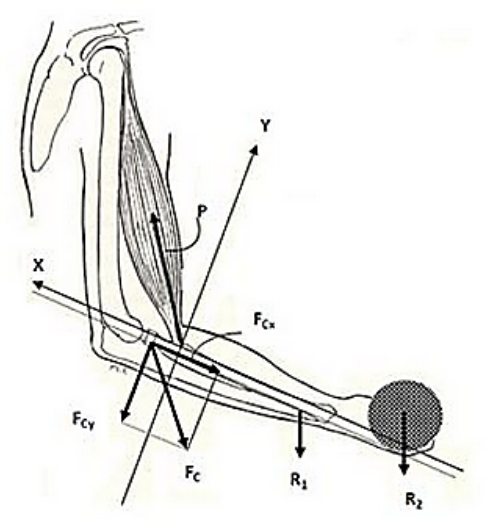


Figura 3. Conjunto de fuerzas actuando sobre una palanca.

Análisis hecho sobre esquema de Luttgens \& Wells, 1982.

1. Potencia (P): Como se ha descrito, es la fuerza voluntaria que se aplica sobre la palanca con alguna intencionalidad. Específicamente, nos interesa la fuerza muscular que se aplica sobre un segmento anatómico con el objetivo de movilizarlo y/o soportar o levantar una carga externa adicional

2. Resistencia (R): Es la fuerza total a vencer. Específicamente, nos interesa la conformada por el peso de la barra o segmento anatómico (R1) y el peso del objeto que levanta (R2). Teniendo en cuenta la tercera ley de Newton (Acción y Reacción), la fuerza ejercida por la carga externa sobre la barra o segmento anatómico, debe ser igual en valor numérico a la fuerza que la barra o segmento anatómico ejerce sobre la carga externa, pero poseen sentidos opuestos.

3. Fuerza de contacto (FC): Es la fuerza de apoyo ejercida por el fulcro sobre la palanca, la cual posee en general dos componentes rectangulares: el componente paralelo al eje mecánico (FCx) y el componente perpendicular al eje mecánico (FCy). En las articulaciones humanas de acuerdo a la tercera ley de Newton o ley de acción y reacción, el primero se opone al componente de la fuerza muscular paralelo al eje mecánico (Herrera, 2001) y, el segundo se opone al componente rotatorio.

\section{Ventaja mecánica en una palanca}

La ventaja mecánica real (VMR) de una máquina se define como el cociente entre la fuerza ejercida por la máquina sobre la carga (Resistencia $=\mathrm{R}$ ) y la fuerza necesaria para operar la máquina (Potencia $=\mathrm{P}$ ). La ventaja mecánica ideal (VMI) se define como el cociente entre el brazo de potencia y el brazo de resistencia. La presencia de fuerzas disipativas expresadas principalmente como fricciones, hacen que la ventaja mecánica real sea siempre menor que la ventaja mecánica ideal $\boldsymbol{V} \boldsymbol{M} \boldsymbol{R}<$ VMP .

Se dice que hay ventaja mecánica solo si ambos cocientes son mayores que 1 , es decir,

Si $\frac{R}{p}>1 \frac{B p}{B r}>1$
En la primera relación de orden en donde $R>P$ significará en un gesto articular que la fuerza muscular aplicada es menor que la carga movilizada.

En la segunda relación de orden $\boldsymbol{B} \boldsymbol{p}>$ $\boldsymbol{B r}$ significará en un gesto articular que la distancia perpendicular de la potencia al apoyo es mayor que la distancia perpendicular de la resistencia al apoyo.

En una palanca en equilibrio la suma de los torques respecto al fulcro es nula y, de acuerdo a la ley de las palancas $\boldsymbol{P} . \boldsymbol{B} \boldsymbol{p}=\boldsymbol{R} . \boldsymbol{B r}$ para sistemas no acelerados como es el caso del gimnasta sostenido sobre las barras y las ejecuciones de un montañista en la escalada, que obedecen a un equilibrio de rotación en el que $\sum \boldsymbol{\tau}_{\boldsymbol{A}}=\mathbf{0}$ Este es el "principio dorado de la mecánica" (Bäumler et al., 1989) que se puede reescribir $\quad \mathbf{P}=\mathbf{R} \frac{\mathbf{B r}}{\mathbf{B p}}$ lo cual significa que manteniendo constante la carga, la fuerza que debe aplicarse varía en forma directamente proporcional con el cociente entre los brazos de resistencia y de potencia. Así, cuando el brazo de resistencia se hace mayor, el cociente entre los brazos aumenta, en consecuencia, la fuerza necesaria para movilizar la carga debe aumentar y, el desplazamiento lineal de la misma es mayor. En cambio, cuando el brazo de resistencia se hace menor, el cociente entre los brazos disminuye, en consecuencia, la fuerza necesaria para movilizar la carga disminuye $y$, el desplazamiento lineal de la misma es menor.

En una palanca que no está en equilibrio, la suma de torques respecto al fulcro es diferente de cero $\sum \boldsymbol{\tau}_{\boldsymbol{A}} \neq \mathbf{0}$ como en el caso de los segmentos anatómicos que rotan aceleradamente en muchos gestos deportivos como los lanzamientos en atletismo y las patadas al balón en fútbol.

\section{Género de palanca}

Hay tres tipos de palanca dependiendo de la ubicación relativa de los puntos críticos (puntos de apoyo y puntos de aplicación de las fuerzas). Para facilitar el análisis, se considera que las líneas de acción de los vectores fuerza (potencia y resistencia) son perpendiculares al eje mecánico de la palanca.

Primer género: Aquella en la cual el punto de apoyo está ubicado entre los puntos de aplicación de la potencia y de la resistencia. Se denomina también inter-apoyo, inter-fija o de 
equilibrio. Pueden ocurrir tres situaciones, las cuales se muestran en la figura 5 :

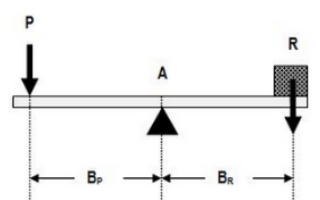

A

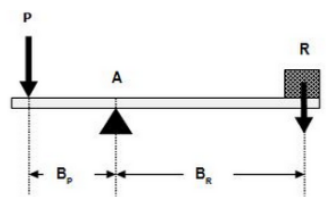

B

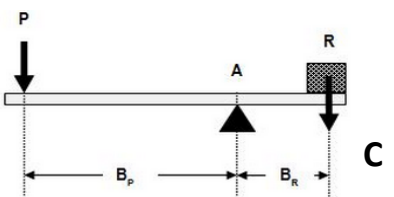

Figura 4. Palanca de primer género y sus variaciones según la ubicación del punto de apoyo

En la figura 4A, El punto de apoyo está ubicado precisamente en la mitad de la distancia que hay entre los puntos de aplicación de $\mathrm{P}$ y de $\mathrm{R}$, en este caso $B \boldsymbol{p}=B r ; p o r$ lo $q u \boldsymbol{e} \frac{B p}{B r}=\mathbf{1}$ y no se obtiene ventaja mecánica.

En la Figura, 4B, El punto de apoyo está ubicado más próximo al punto de aplicación de $P$, en este caso, $\boldsymbol{B p}<\boldsymbol{B r}$; por lo que $\frac{\boldsymbol{B} \boldsymbol{p}}{\boldsymbol{B r}}<\mathbf{1}$; no se obtiene ventaja mecanica, se debe realizar mas fuerza para levantar la carga que en la situación $4 \mathrm{~A}$.

En la figura $5 \mathrm{C}$, El punto de apoyo está ubicado más próximo al punto de aplicación de $\mathrm{R}$, en este caso, $\boldsymbol{B} \boldsymbol{p}>\boldsymbol{B r}$, por lo que, $\frac{\boldsymbol{B} \boldsymbol{p}}{\boldsymbol{B} \boldsymbol{r}}>\mathbf{1}$, obteniéndose ventaja mecánica.

Segundo género: Aquella en la cual el punto de aplicación de la resistencia está ubicado entre los puntos de aplicación de la potencia y del apoyo. Se denomina también inter-resistente 0 favorable a la fuerza, dado que hay que aplicar menos fuerza para levantar la carga. Pueden ocurrir tres situaciones, las cuales se muestran en la figura 5.
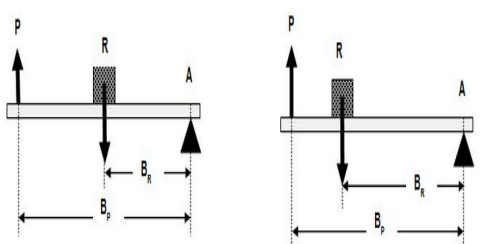

A

B

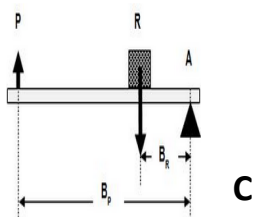

Figura 5. Palanca de segundo género y sus variaciones según la ubicación de la resistencia.

En la figura $5 \mathrm{~A}$, El punto de aplicación de $\mathrm{R}$ está ubicado precisamente en la mitad de la distancia que hay entre los puntos de aplicación de $\mathrm{P}$ y el apoyo, en este caso: $\boldsymbol{B} \boldsymbol{p}=\mathbf{2 B} \boldsymbol{R}$, por lo que, $\frac{B p}{B r}=\frac{2 B p}{B r}=2>1$, y se obtiene una ventaja mecánica, cuyo valor es de 2.

En la figura 5B, El punto de aplicación de R está ubicado más próximo al punto de aplicación de $P$, en este caso $B r<B p<2 B r$ por lo que, $\frac{B p}{B r}>1$, y se obtiene una ventaja mecánica cuyo valor es mayor que 1 y menor que 2, es decir, se debe aplicar más fuerza que en el caso $5 \mathrm{~A}$ para levantar la misma carga.

En la Figura 5C, El punto de aplicación de R está ubicado más próximo al punto de apoyo: $B p>2 B r$, por lo que, $\frac{B p}{B r}>1$, y se obtiene una ventaja mecánica cuyo valor es mayor que 2, es decir, para levantar la misma carga se aplica menos fuerza que en las situaciones $5 \mathrm{~A}$ y $5 \mathrm{~B}$.

Tercer género: Aquella en la cual el punto de aplicación de la potencia está ubicado entre los puntos de aplicación de la resistencia y el apoyo. Se denomina también inter-potente, de velocidad o desfavorable a la fuerza, dado que hay que aplicar más fuerza para levantar la carga. Pueden ocurrir tres situaciones, las cuales se muestran en la figura 7 . 

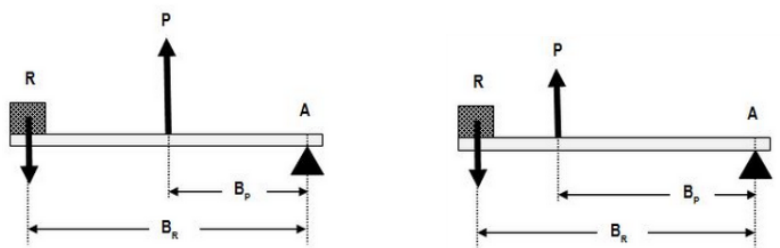

A

B

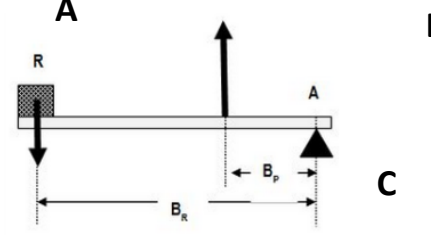

Figura 6. Palanca de tercer género y sus variaciones según la ubicación de la potencia.

En la figura $6 \mathrm{~A}$, El punto de aplicación de P está ubicado precisamente en la mitad de la distancia que hay entre los puntos de aplicación de $\mathrm{R}$ y del apoyo, en este caso, $\quad \boldsymbol{B} \boldsymbol{p}=$ $\frac{B r}{2}$, por lo que, $\frac{B p}{B r}=\frac{\frac{B r}{2}}{B r}=\frac{1}{2}=0,5<1, y$ no se obtiene ventaja mecánica.

En la figura 6B, El punto de aplicación de P está ubicado más próximo al punto de aplicación de $\mathrm{R}$, en este caso $\frac{B r}{2}<B p<B r$, por lo que, $\frac{B p}{B r}<1$, y no se obtiene ventaja mecánica ya que el valor numérico del cociente es mayor que 0,5 pero menor que 1, en esta situación se aplica menos fuerza que en el caso $6 \mathrm{~A}$ para levantar la misma carga.

En la figura 6C, El punto de aplicación de P está ubicado más próximo al punto de apoyo, en este caso $\boldsymbol{B} \boldsymbol{p}<\frac{\boldsymbol{B r}}{2}$, por lo que, $\frac{B \boldsymbol{p}}{\boldsymbol{B r}}<\frac{1}{2}$ y no se obtiene ventaja mecanica ya que el valor numérico del cociente es menor que 0,5 (por tanto menor que 1). En esta situación se aplica más fuerza que en los casos $6 \mathrm{~A}$ y $6 \mathrm{~B}$ para levantar la misma carga.

Observamos que en las palancas de tercer género siempre ocurre que $\boldsymbol{B} \boldsymbol{p}<\boldsymbol{B r}$ por lo que nunca obtienen ventaja mecánica.

La mayoría de palancas en el cuerpo humano son de tercer género, esto significa que no está construido para obtener ventaja mecánica levantando cargas, sino más bien para generar desplazamientos de los segmentos. En la mayoría de las ocasiones, el brazo de potencia en el sistema osteomuscular humano, es más corto que el brazo de resistencia. Así, las palancas anatómicas tienden a favorecer la velocidad y el grado de movimiento (amplitud) a expensas del esfuerzo (Luttgens y Wells, 1982). "Los músculos esqueléticos desarrollan fuerzas mayores que las cargas que soportan; sin embargo, las cargas pueden moverse mucho más de lo que se contrae el músculo" (Piña, 2002). Acciones cotidianas como las extensiones y flexiones de rodilla durante la marcha, levantar un objeto flexionando el codo y patear un balón extendiendo la rodilla, son ejemplos simples de gestos que requieren de una gran fuerza muscular (mayor que la carga levantada) pero que se caracterizan por un desplazamiento importante de la parte distal del segmento anatómico movilizado. El hecho de levantar flexionando el codo con el antebrazo supinado, un alimento para llevarlo a la boca, implica por parte del bíceps braquial la ejecución de una fuerza más de 6 veces superior (Tuszynski, 2001) a la carga levantada (peso del segmento anatómico antebrazomuñeca-mano más el peso del alimento levantado, por liviano que sea), con un brazo de potencia muy inferior al brazo de resistencia, indicando ausencia de ventaja mecánica, pero con un efecto compensador importante expresado en el desplazamiento de la mano para cumplir eficazmente con el propósito del gesto. Si el punto de inserción del bíceps no estuviera ubicado en la tuberosidad radial, sino que fuera tan distal como para quedar más alejado del codo que el baricentro de la carga, de modo que produjera ventaja mecánica, disminuiría bastante la fuerza que debería ejercer el músculo bíceps (incluso de manera razonable su sección transversal sería menor), pero la reducción del desplazamiento de la mano, generaría gastos energéticos importantes en la musculatura cervical y torácica que tendrían que actuar en una búsqueda forzada del alimento. En este sentido, cuando se activan en contracción concéntrica, los músculos de origen lejano e inserción cercana con respecto al fulcro articular, se producen grandes amplitudes de movimiento (Grosser et al., 1991), es decir, un movimiento muy pequeño del extremo corto de la palanca queda magnificado en proporción directa a la longitud de la palanca que se está movilizando (Gowitzke and Milner, 1999). En cambio, cuando se activan en contracción concéntrica, los músculos de origen cercano e inserción 
lejana, las amplitudes de movimiento son claramente inferiores.

La denominada por muchos "desventaja mecánica" de las palancas anatómicas corporales humanas, por el hecho de ser el cociente entre los brazos de potencia y de resistencia, menor o igual que 1 , es coherente con el hecho de cierta delgadez relativa de los músculos para ubicarse paralelamente al hueso y con la característica contráctil (concéntrica) del músculo estriado que lo lleva a adquirir una longitud un $30 \%$ menor de la original (MacDonald, 1989). Si obtuvieran ventaja mecánica, con un cociente mayor que 1 , tal como se explicó anteriormente, los movimientos articulares serían ineficaces.

El estudio del movimiento corporal humano analizado como un sistema de palancas permite entender como los trastornos y lesiones del sistema musculo-esquelético terminan en última instancia afectando el normal funcionamiento del sistema de palancas al alterar uno o varios componentes del sistema, tal como se muestra en las figuras 7,8 y 9 .

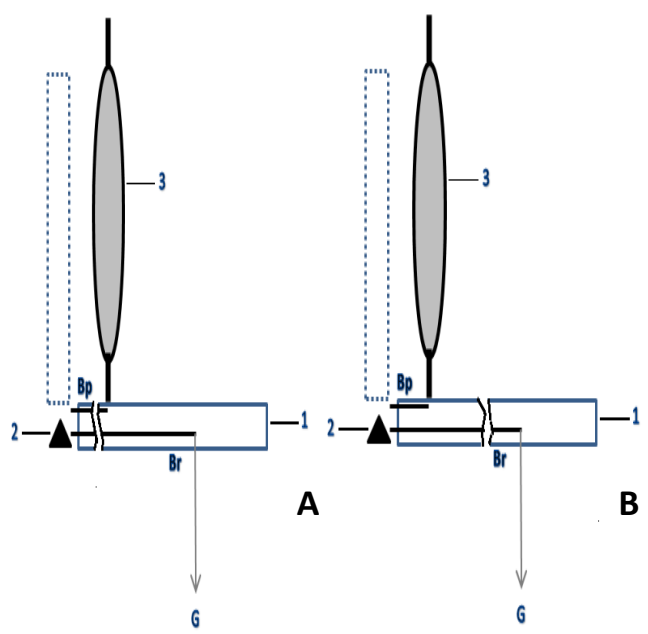

\begin{tabular}{|l|l|}
\hline 1. Hueso & 1. Palanca \\
\hline 2. Articulación & 2. Punto de apoyo. \\
\hline 3. Musculo & 3. Vector fuerza. \\
\hline 4. Gravedad. & 4. Vector Gravedad \\
\hline \multicolumn{2}{|c|}{ Bp: Brazo de palanca } \\
\hline \multicolumn{2}{|c|}{ Br : Brazo de resistencia } \\
\hline
\end{tabular}

Figura 7. Ejemplo de alteración en la palanca en un sistema de tercer género.
En una palanca de tercer género tal como la que se muestra en la figura $7 A$, se puede observar que una alteración estructural ósea como la sucedida en una fractura que compromete al hueso entre la articulación (el fulcro) y el sitio de inserción del tendón del musculo (punto de aplicación de la fuerza) compromete la continuidad del brazo de potencia $(\mathrm{Bp})$ y del brazo de resistencia $(\mathrm{Br})$ de la palanca, tales alteraciones se pueden presentar en las fracturas de la cabeza radial 0 una fractura metafisiaria del cubito, para el caso específico del ejemplo. En la figura 7B la fractura se ubica después del punto de aplicación de la fuerza lo que generará una alteración del brazo de palanca de la resistencia como la que sucederá para el caso particular en una fractura en la diáfisis del radio o del cubito.

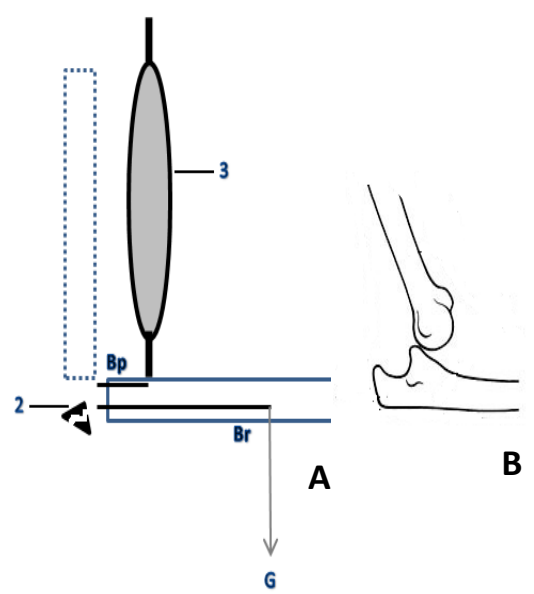

\begin{tabular}{|l|l|}
\hline 1. Hueso & 1. Palanca \\
\hline 2. Articulación & 2. Punto de apoyo. \\
\hline 3. Musculo & 3. Vector fuerza. \\
\hline 4. Gravedad. & 4. Vector Gravedad \\
\hline \multicolumn{2}{|c|}{ Bp: Brazo de palanca } \\
\hline \multicolumn{2}{|c|}{ Br : Brazo de resistencia } \\
\hline
\end{tabular}

Figura 8. Ejemplo de alteración en el apoyo en un sistema de palanca de tercer género.

En la figura 8A, se da un ejemplo de alteración del apoyo o fulcro en un sistema de palancas de tercer género. La alteración compromete el sitio donde se produce el giro de la palanca (el movimiento del segmento corporal), este tipo de lesiones se presentan en las estructuras osteo- 
articulares por pérdida parcial o total en la relación de las superficies articulares del hueso, tal como sucede en las luxaciones $y$ subluxaciones articulares del codo, figura 8B.
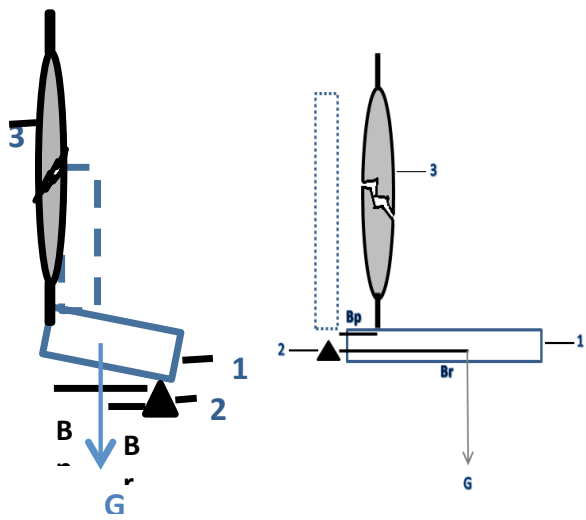

\begin{tabular}{l|l|}
\hline 1. Hueso & 1. Palanca \\
\hline 2. Articulación & 2. Punto de apoyo. \\
\hline 3. Musculo & 3. Vector fuerza. \\
\hline 4. Gravedad. & 4. Vector Gravedad \\
\hline \multicolumn{2}{|c|}{ Bp: Brazo de palanca } \\
\hline \multicolumn{2}{|c|}{ Br : Brazo de resistencia } \\
\hline
\end{tabular}

Figura 9. Ejemplo de alteración en la fuerza en un sistema de palanca de segundo y tercer género.

En la figura 9, se da un ejemplo de una alteración en el musculo, lo cual compromete al vector fuerza (motor) del sistema de palancas, este tipo de alteraciones pueden presentarse por fallas estructurales del musculo como rupturas parciales 0 totales, pero también por alteraciones en la motoneurona 0 en la unidad motora del respectivo musculo.

Como se puede observar el movimiento corporal humano desde los más simple hasta los más complejo pueden interpretarse como el resultado de la confrontación de las fuerzas internas corporales con las fuerzas externas del medio ambiente, las cuales pueden ser interpretadas y comprendidas desde la física mecánica, en especial como un sistema de palancas y como un sistema de momentos de fuerza, los cuales están en constante interacción para lograr el rendimiento adecuado del individuo, además el estudio del movimiento corporal desde estas perspectivas permite la comprensión de las consecuencias mecánicas de las alteraciones de las estructuras del sistema muscular, óseo y articular del individuo.

\section{REFERENCIAS}

Aguado Jódar, Xavier. Eficacia y técnica deportiva. Análisis del movimiento humano. $1^{\text {a }}$ ed. Barcelona: INDE Publicaciones. 1993

Arcila, Juan; Cardona, Donaldo y Giraldo, Juan. Abordaje físico-matemático del gesto articular. EFDeportes.com. Revista digital. Buenos Aires, $\begin{array}{llll}\text { Año } & 17, & N^{0} & 171 .\end{array}$ 2012.http://www.efdeportes.com/efd171/abordaj e-fisico-matematico-del-gesto-articular.htm

Bäumler, Günter y Schneider, Klaus. Biomecánica deportiva. Fundamentos para el estudio y la práctica. Barcelona: Martínez Roca, S.A. 1989.

Bueche, Frederick J. Física general. $3^{\mathrm{a}}$ ed. México: McGraw-Hill. 1991.

Calais-Germain, Blandine. Anatomía para el movimiento. Tomo I. Introducción al análisis de las técnicas corporales. Barcelona: La liebre de Marzo. 2010.

Donskoi, D. Biomecánica con fundamentos de la técnica deportiva. La Habana: Pueblo y Educación. 1982.

Enoka, Roger M. Neuromechanics of human movement. $3^{\mathrm{a}}$ ed. Champaign: Human Kinetics. 2002.

Fucci, Sergio; Benigni, Mario y Fornasari, Vittorio. Biomecánica del aparato locomotor aplicada al acondicionamiento muscular. $3^{\mathrm{a}}$ ed. Barcelona: Harcourt Brace. 1998.

Gowitzke, Barbara y Milner, Morris. El cuerpo y sus movimientos. Bases científicas. $3^{a}$ ed. Barcelona: Paidotribo. 1999. 
Grosser, Manfred. Heike, Hermann; Tusker, Ferdinand y Zintl, Fritz. El movimiento deportivo. Bases anatómicas y biomecánicas. Barcelona: Martínez roca S. A. 1991.

Guillén del Castillo, Manuel y Linares Girela, Daniel. Bases biológicas y fisiológicas del movimiento humano. Madrid: Médica Panamericana S.A. 2002.

Gutiérrez Dávila, Marcos. Biomecánica deportiva. $1^{\text {a }}$ reimpresión. Madrid: Síntesis. 1999.

Herrera, Miguel Ángel. Biofísica, Geofísica, Astrofísica. Para qué sirve la Física. México: Ediciones Científicas Universitarias, Universidad Nacional Autónoma de México. 2001.

Hochmuth, Gerhard. Biomecánica de los movimientos deportivos. $1^{\text {a }}$ ed. Madrid: DONCEL, Instituto Nacional de Educación Física. 1973.

Luttgens, K. Wells, K. Kinesiology: Scientific Basis of human motion. Philadelphia. Saunders College publishing, 1982.

MacDonald, Simon y Burns, Desmond. Física. Wilmington, Delaware, E. U. A.: Addison-Wesley Iberoamericana, S. A.
Marion, Jerry B. and Hornyak, William, F. Physics for science and engineering, Part 1. University of Maryland. New York: Saunders College Publishing. 1982.

Piña Barba, María Cristina. La física en medicina. $3^{\mathrm{a}}$ ed. México: La Ciencia para todos, 37. 2002.

Rasch, Philip y Burke, Roger. Kinesiología y anatomía aplicada. $6^{a}$ ed. Buenos Aires: El Ateneo. 1986.

Vander, F. Analyse des mouvements du corps humain. Desoer, Liège. 1961.

Vogel, Steven. Comparative biomechanics. Life's physical world. Princeton University Press: 2003.

Tuszynski, Jack A. and Dixon, John M. Biomedical applications of introductory physics. New York: John Wiley \& Sons. Inc. 2002.

Zatsiorski, V y Donskoi, D. Biomecánica de los ejercicios físicos. $1^{a}$ reimpresión. La Habana: Pueblo y Educación. 1990. 